\title{
The association between first and second wave COVID-19 mortality in Italy
}

\author{
Marco Vinceti ${ }^{1,2^{*}}$ (D, Tommaso Filippini ${ }^{1} \mathbb{B}$, Kenneth J. Rothman ${ }^{2,3}$ (D), Silvia Di Federico ${ }^{1}$ and Nicola Orsini ${ }^{4}$ (D)
}

\begin{abstract}
Background: The relation between the magnitude of successive waves of the COVID-19 outbreak within the same communities could be useful in predicting the scope of new outbreaks.

Methods: We investigated the extent to which COVID-19 mortality in Italy during the second wave was related to first wave mortality within the same provinces. We compared data on province-specific COVID-19 2020 mortality in two time periods, corresponding to the first wave (February 24-June 30, 2020) and to the second wave (September 1-December 31, 2020), using cubic spline regression.

Results: For provinces with the lowest crude mortality rate in the first wave (February-June), i.e. < 22 cases/ 100,000/month, mortality in the second wave (September-December) was positively associated with mortality during the first wave. In provinces with mortality greater than 22/100,000/month during the first wave, higher mortality in the first wave was associated with a lower second wave mortality. Results were similar when the analysis was censored at October 2020, before the implementation of region-specific measures against the outbreak. Neither vaccination nor variant spread had any role during the study period.
\end{abstract}

Conclusions: These findings indicate that provinces with the most severe initial COVID-19 outbreaks, as assessed through mortality data, faced milder second waves.

Keywords: COVID-19, Epidemiology, Mortality, Public health, SARS-CoV-2, Waves

\section{Background}

The COVID-19 pandemic in many countries has been characterized by waves of infection. These recurrent local exacerbations of the pandemic present an opportunity to study the spread of the virus within a population. Key features of the pandemic are still not well understood, such as the susceptibility of the population to subsequent waves after the first outbreak, the threshold for herd immunity, the role of superspreaders [1-7] as well as of meteorological and environmental factors [8-11].

\footnotetext{
*Correspondence: marco.vinceti@unimore.it

${ }^{1}$ Environmental, Genetic and Nutritional Epidemiology Research Center (CREAGEN), Section of Public Health, Department of Biomedical, Metabolic and Neural Sciences, University of Modena and Reggio Emilia, Modena, Italy ${ }^{2}$ Department of Epidemiology, Boston University School of Public Health, Boston, MA, US

Full list of author information is available at the end of the article
}

We compared the two COVID-19 waves within Italy, where the geographical distribution of the SARS-CoV-2 infection spread and the COVID-19 incidence was uneven during the first wave of the pandemic [12]. From an analysis within provinces of the official case counts during the first and second wave of SARS-CoV-2 infection, we found evidence of an association in the occurrence between the two periods. When incidence in the first wave was low $(<500$ cases/100,000/day), the second wave incidence tended to be positively correlated, whereas a high first-wave incidence was strongly and inversely correlated with the second wave incidence. This observation raised the possibility that greater spread at the beginning of the pandemic could have induced some protection at the population level, resulting in a milder second wave, despite low levels (10-20\%) of anti-SARS-

(c) The Author(s). 2021 Open Access This article is licensed under a Creative Commons Attribution 4.0 International License, which permits use, sharing, adaptation, distribution and reproduction in any medium or format, as long as you give appropriate credit to the original author(s) and the source, provide a link to the Creative Commons licence, and indicate if changes were made. The images or other third party material in this article are included in the article's Creative Commons licence, unless indicated otherwise in a credit line to the material. If material is not included in the article's Creative Commons licence and your intended use is not permitted by statutory regulation or exceeds the permitted use, you will need to obtain permission directly from the copyright holder. To view a copy of this licence, visit http://creativecommons.org/licenses/by/4.0/ The Creative Commons Public Domain Dedication waiver (http://creativecommons.org/publicdomain/zero/1.0/) applies to the data made available in this article, unless otherwise stated in a credit line to the data. 
CoV-2 antibody seroprevalence even in the areas most severely hit.

One problem with our earlier finding is that the incidence of SARS-CoV-2 infection is difficult to measure. It depends on the severity of the clinical course, the availability of molecular tests and the policy underlying their use at the population level. In Italy, for instance, the implementation of nasopharyngeal swabs and molecular tests to detect SARS-CoV-2 infection in the first wave was restricted primarily to symptomatic patients with suspected COVID-19. In the summer of 2020, however, the public-health policy was modified to extend testing to contacts of cases and to individuals without clinical symptoms, nearly tripling the number of daily molecular tests from the first to the second wave time periods [13]. An additional change of policy, starting on November 6 , 2020, during the second wave, was the mandatory adoption of differential area-specific public-health measures $[14,15]$.

The area-specific relation between the two waves may be better assessed by a more objective measures of COVID-19 spread such as mortality rate, instead of incidence rates that are subject to swab testing policy and availability. Recently, COVID-19 death data for Italy in 2020 have become available with greater geographic detail, with figures available for the 107 provinces rather than the 20 larger regions within Italy. We used these mortality data to revisit the relation of the dynamics of the first and second waves of the outbreak.

\section{Methods}

\section{Study population and outcome}

We used data made freely available from public sources. We accessed 2020 monthly province-specific COVID-19 deaths as reported by the Italian National Institute of Statistics - ISTAT [16]. Provinces are the administrative entities that are intermediate between smaller municipalities and larger regions within Italy. Within 2020, we examined two time frames: we considered the first wave to be the period from the pandemic onset (February 24) to June 30, when the number of new infection cases had substantially dropped after the March-April peak. From July to the end of August the number of new cases remained low, mostly related to screening of subjects returning from vacation with swab testing. Cases increased again beginning in early September. Therefore, we considered the second wave to be the period from September 1 to December 31, 2020. During these periods, neither circulation of virus variants nor implementation of SARS-CoV-2 vaccination at the population level had yet started in Italy. Only a handful of Italians had been vaccinated by the end of December, but large scale administration started in January 2021 [17, 18].
Based on these data and the Italian population at January 1, 2020, and January 1, 2021 available at the ISTAT website [19], we computed wave-specific COVID-19 mortality during the first and second waves. For the former period, we used as reference population data the provincial population size as of January 1, 2020. For the second period and for the overall 2020 mortality we averaged the population size at January 1, 2020 and January 1, 2021 for each province. We retrieved the seroprevalence data for each Italian region and province determined through a national survey made available by ISTAT and the National Ministry of Health with reference to the period May 25, 2020 through July 15, 2020 [20].

\section{Data analysis}

We examined the relation between first and second wave province-specific crude mortality rates from COVID-19 by modeling the mean mortality rate using a restricted cubic spline regression [21], a model that fits a curvilinear pattern to the data as previously reported [22-24]. In particular, we fitted a restricted cubic spline model that weighted provincial data by population size, performing both a crude analysis and adjusting for potential confounders. Variables included in the model were aging index (i.e. a ratio between resident population aged $\geq 65$ years and those aged $\leq 14$ years), percentage commuting outside the municipality of residence on a daily basis, and percentage of dwellings occupied by only one resident (available at a provincial level from the National Institute of Statistics [19]). In the regression model, we used three knots at fixed percentiles (10th, 50th and 90th) of the first wave distribution, and computed a pointwise $95 \%$ confidence interval $(\mathrm{CI})[21,25]$. We used 'mkspline', 'regress', and 'xbrcsplinei' routines of Stata software (v17.0, College Station-TX, 2021) for all analyses.

\section{Results}

Table 1 and Fig. 1 lists province-specific mortality rates in the investigated periods. National cumulative mortality (cumulative incidence of death) during the first wave averaged 58.2/100,000 persons, ranging from 6.5/100,000 persons in the Southern Italy, Basilicata region, to 165/ 100,000 persons in Northern Italy, Lombardy region. Corresponding figures for the second wave period were $67.6 / 100,000$ persons for the national average, ranging from 19.4/100,000 in the Calabria region to $192 / 100,000$ persons in the Aosta Valley region. In particular, seven provinces, five of the in Lombardy region (Bergamo, Brescia, Cremona, Lodi, and Pavia) and two in EmiliaRomagna region (Parma and Piacenza) experienced the highest mortality rates during the first wave. Second wave mortality was lower than first wave, always $\leq 100$ / 
Table 1 Number of SARS-CoV-2 cases, COVID-19 deaths and COVID-19 mortality rates (deaths/100,000/wave timeframe) in the 1st and 2nd waves in 2020 divided by province

\begin{tabular}{|c|c|c|c|c|c|c|c|c|c|c|c|}
\hline $\begin{array}{l}\text { Province/ } \\
\text { Region }\end{array}$ & $\begin{array}{l}\text { Population } \\
\text { Jan 1, } 2020\end{array}$ & $\begin{array}{l}\text { Population } \\
\text { Jan 1, } 2021\end{array}$ & $\begin{array}{l}\text { Cases } \\
1 \text { st } \\
\text { wave }\end{array}$ & $\begin{array}{l}\text { Cases } \\
\text { 2nd } \\
\text { wave }\end{array}$ & $\begin{array}{l}\text { Seroprev. } \\
\text { (\%) }\end{array}$ & $\begin{array}{l}\text { Deaths } \\
\text { 1st wave }\end{array}$ & $\begin{array}{l}\text { Deaths } \\
\text { 2nd } \\
\text { wave }\end{array}$ & $\begin{array}{l}\text { Mortality } \\
\text { 1st wave }\end{array}$ & $\begin{array}{l}\text { Mortality } \\
\text { 2nd wave }\end{array}$ & $\begin{array}{l}\text { All } \\
\text { Deaths }\end{array}$ & $\begin{array}{l}\text { Overall } \\
\text { Mortality }\end{array}$ \\
\hline $\begin{array}{l}\text { Aosta } \\
\text { Valley }\end{array}$ & 125,501 & 123,895 & 1195 & 5771 & 3.72 & 145 & 239 & 116 & 192 & 384 & 308 \\
\hline Aosta & 125,501 & 123,895 & 1195 & 5771 & 3.72 & 145 & 239 & 116 & 192 & 384 & 308 \\
\hline Lombardy & $10,103,969$ & $9,966,992$ & 91,813 & 368,273 & 7.35 & 16,633 & 8321 & 165 & 82.9 & 25,120 & 250 \\
\hline Bergamo & $1,116,384$ & $1,099,621$ & 14,375 & 12,873 & 24.3 & 3137 & 193 & 281 & 17.4 & 3347 & 302 \\
\hline Brescia & $1,268,455$ & $1,247,583$ & 15,626 & 25,468 & 7.63 & 2686 & 422 & 212 & 33.5 & 3117 & 248 \\
\hline Como & 603,828 & 594,671 & 4093 & 29,531 & 2.00 & 587 & 794 & 97.2 & 133 & 1388 & 232 \\
\hline Cremona & 358,347 & 351,698 & 6612 & 7664 & 19.7 & 1130 & 123 & 315 & 34.6 & 1261 & 355 \\
\hline Lecco & 337,087 & 332,593 & 2831 & 10,303 & 6.66 & 481 & 236 & 143 & 70.5 & 724 & 216 \\
\hline Lodi & 230,607 & 225,885 & 3570 & 6936 & 7.10 & 679 & 140 & 294 & 61.3 & 826 & 362 \\
\hline Mantua & 411,062 & 403,585 & 3496 & 12,260 & 6.57 & 684 & 288 & 166 & 70.7 & 975 & 239 \\
\hline Milan & $3,279,944$ & $3,249,821$ & 24,379 & 147,720 & 3.95 & 4252 & 3197 & 130 & 97.9 & 7509 & 230 \\
\hline $\begin{array}{l}\text { Monza/ } \\
\text { Brianza }\end{array}$ & 878,267 & 867,421 & 5772 & 42,090 & 4.52 & 979 & 895 & 112 & 103 & 1884 & 216 \\
\hline Pavia & 546,515 & 534,951 & 5568 & 18,869 & 5.95 & 1241 & 543 & 227 & 100 & 1806 & 334 \\
\hline Sondrio & 180,941 & 179,234 & 1584 & 6954 & 5.30 & 212 & 201 & 117 & 112 & 415 & 230 \\
\hline Varese & 892,532 & 879,929 & 3907 & 47,605 & 1.71 & 565 & 1289 & 63.3 & 145 & 1868 & 211 \\
\hline Veneto & $4,907,704$ & $4,852,453$ & 18,937 & 227,276 & 1.92 & 2028 & 4960 & 41.3 & 102 & 7079 & 145 \\
\hline Belluno & 201,972 & 199,599 & 1191 & 13,369 & 1.88 & 114 & 330 & 56.4 & 164 & 445 & 222 \\
\hline Padua & 939,672 & 929,520 & 3954 & 41,651 & 2.32 & 318 & 608 & 33.8 & 65.1 & 946 & 101 \\
\hline Rovigo & 233,386 & 229,652 & 444 & 6932 & 2.39 & 36 & 204 & 15.4 & 88.1 & 240 & 104 \\
\hline Treviso & 888,309 & 878,070 & 2673 & 45,715 & 1.89 & 322 & 783 & 36.2 & 88.7 & 1112 & 126 \\
\hline Venice & 851,663 & 842,942 & 2682 & 35,612 & 1.68 & 299 & 863 & 35.1 & 102 & 1185 & 140 \\
\hline Verona & 930,339 & 922,291 & 5127 & 44,073 & 2.23 & 586 & 1195 & 63.0 & 129 & 1789 & 193 \\
\hline Vicenza & 862,363 & 850,379 & 2866 & 39,924 & 1.33 & 353 & 977 & 40.9 & 114 & 1362 & 159 \\
\hline $\begin{array}{l}\text { Emilia- } \\
\text { Romagna }\end{array}$ & $4,467,118$ & $4,445,549$ & 28,061 & 137,052 & 2.90 & 4353 & 3431 & 97.4 & 77.0 & 7825 & 176 \\
\hline Bologna & $1,017,806$ & $1,019,539$ & 5229 & 32,314 & 2.33 & 732 & 936 & 71.9 & 91.9 & 1681 & 165 \\
\hline Ferrara & 344,840 & 341,967 & 1044 & 7886 & 0.72 & 173 & 222 & 50.2 & 64.6 & 396 & 115 \\
\hline Forlì-Cesena & 394,833 & 393,556 & 1740 & 10,213 & 1.04 & 196 & 164 & 49.6 & 41.6 & 362 & 91.8 \\
\hline Modena & 707,292 & 704,672 & 3873 & 25,945 & 1.10 & 480 & 601 & 67.9 & 85.1 & 1085 & 154 \\
\hline Parma & 453,930 & 453,604 & 3657 & 8701 & 5.84 & 901 & 215 & 199 & 47.4 & 1119 & 247 \\
\hline Piacenza & 287,236 & 284,075 & 4428 & 10,187 & 9.54 & 956 & 252 & 333 & 88.2 & 1213 & 425 \\
\hline Ravenna & 389,634 & 386,309 & 1030 & 11,337 & 1.18 & 81 & 430 & 20.8 & 111 & 514 & 133 \\
\hline $\begin{array}{l}\text { Reggio } \\
\text { nell'Emilia }\end{array}$ & 531,751 & 526,349 & 4913 & 18,248 & 4.45 & 581 & 306 & 109 & 57.8 & 891 & 168 \\
\hline Rimini & 339,796 & 335,478 & 2147 & 12,221 & 2.79 & 253 & 305 & 74.5 & 90.3 & 564 & 167 \\
\hline Piedmont & $4,341,375$ & $4,273,210$ & 30,989 & 162,730 & 3.45 & 4029 & 3537 & 92.8 & 82.1 & 7583 & 176 \\
\hline Alessandria & 419,037 & 411,922 & 4063 & 13,240 & 2.08 & 659 & 470 & 157 & 113 & 1134 & 273 \\
\hline Asti & 213,216 & 209,648 & 1874 & 7960 & 2.13 & 249 & 217 & 117 & 103 & 466 & 220 \\
\hline Biella & 174,384 & 171,838 & 1046 & 5748 & 6.59 & 194 & 112 & 111 & 64.7 & 306 & 177 \\
\hline Cuneo & 586,568 & 582,353 & 2862 & 24,081 & 0.87 & 373 & 494 & 63.6 & 84.5 & 869 & 149 \\
\hline Novara & 368,040 & 362,199 & 2792 & 12,443 & 5.21 & 367 & 272 & 99.7 & 74.5 & 642 & 176 \\
\hline
\end{tabular}


Table 1 Number of SARS-CoV-2 cases, COVID-19 deaths and COVID-19 mortality rates (deaths/100,000/wave timeframe) in the 1st and 2 nd waves in 2020 divided by province (Continued)

\begin{tabular}{|c|c|c|c|c|c|c|c|c|c|c|c|}
\hline $\begin{array}{l}\text { Province/ } \\
\text { Region }\end{array}$ & $\begin{array}{l}\text { Population } \\
\text { Jan 1, } 2020\end{array}$ & $\begin{array}{l}\text { Population } \\
\text { Jan 1, } 2021\end{array}$ & $\begin{array}{l}\text { Cases } \\
1 \text { st } \\
\text { wave }\end{array}$ & $\begin{array}{l}\text { Cases } \\
\text { 2nd } \\
\text { wave }\end{array}$ & $\begin{array}{l}\text { Seroprev. } \\
\text { (\%) }\end{array}$ & $\begin{array}{l}\text { Deaths } \\
\text { 1st wave }\end{array}$ & $\begin{array}{l}\text { Deaths } \\
\text { 2nd } \\
\text { wave }\end{array}$ & $\begin{array}{l}\text { Mortality } \\
\text { 1st wave }\end{array}$ & $\begin{array}{l}\text { Mortality } \\
\text { 2nd wave }\end{array}$ & $\begin{array}{l}\text { All } \\
\text { Deaths }\end{array}$ & $\begin{array}{l}\text { Overall } \\
\text { Mortality }\end{array}$ \\
\hline Turin & $2,252,379$ & $2,212,996$ & 15,889 & 87,788 & 3.58 & 1844 & 1712 & 81.9 & 76.7 & 3561 & 160 \\
\hline $\begin{array}{l}\text { Verbano- } \\
\text { Cusio-Ossola }\end{array}$ & 157,455 & 155,065 & 1140 & 5515 & 9.05 & 132 & 122 & 83.8 & 78.1 & 254 & 163 \\
\hline Vercelli & 170,296 & 167,189 & 1323 & 5955 & 3.52 & 211 & 138 & 124 & 81.8 & 351 & 208 \\
\hline $\begin{array}{l}\text { Trentino- } \\
\text { South Tyrol }\end{array}$ & $1,074,819$ & $1,078,460$ & 7502 & 43,303 & 9.93 & 693 & 1041 & 64.5 & 96.7 & 1734 & 161 \\
\hline Bolzano & 532,080 & 533,715 & 2639 & 26,559 & 2.95 & 288 & 504 & 54.1 & 94.6 & 792 & 149 \\
\hline Trento & 542,739 & 544,745 & 4863 & 16,744 & 3.42 & 405 & 537 & 74.6 & 98.8 & 942 & 173 \\
\hline $\begin{array}{l}\text { Friuli- } \\
\text { Venezia } \\
\text { Giulia }\end{array}$ & $1,211,357$ & $1,198,753$ & 3308 & 45,651 & 1.02 & 362 & 1426 & 29.9 & 118 & 1794 & 149 \\
\hline Gorizia & 139,206 & 136,809 & 216 & 5904 & 0.12 & 5 & 104 & 3.6 & 75.4 & 109 & 79.0 \\
\hline Pordenone & 312,619 & 309,058 & 702 & 9792 & 1.88 & 68 & 291 & 21.8 & 93.6 & 360 & 116 \\
\hline Trieste & 233,276 & 229,470 & 1393 & 9107 & 0.59 & 209 & 270 & 89.6 & 117 & 483 & 209 \\
\hline Udine & 526,256 & 523,416 & 997 & 20,848 & 0.93 & 80 & 761 & 15.2 & 145 & 842 & 160 \\
\hline Liguria & $1,543,127$ & $1,509,805$ & 9473 & 46,958 & 3.24 & 1563 & 1276 & 101 & 86.3 & 2851 & 187 \\
\hline Genoa & 835,829 & 816,916 & 5573 & 29,304 & 3.61 & 943 & 853 & 113 & 103 & 1802 & 218 \\
\hline Imperia & 213,919 & 208,585 & 1494 & 4806 & 2.39 & 231 & 79 & 108 & 37.4 & 312 & 148 \\
\hline La Spezia & 219,196 & 215,538 & 860 & 7142 & 1.89 & 159 & 189 & 72.5 & 86.9 & 349 & 161 \\
\hline Savona & 274,183 & 268,766 & 1546 & 5706 & 3.83 & 230 & 155 & 83.9 & 57.1 & 388 & 143 \\
\hline Tuscany & $3,722,729$ & $3,668,333$ & 9779 & 108,429 & 0.90 & 1088 & 2491 & 29.2 & 67.4 & 3604 & 97.5 \\
\hline Arezzo & 341,766 & 336,870 & 676 & 9779 & 1.23 & 47 & 168 & 13.8 & 49.5 & 218 & 64.2 \\
\hline Florence & $1,004,298$ & 986,001 & 3192 & 29,864 & 0.53 & 401 & 839 & 39.9 & 84.3 & 1249 & 126 \\
\hline Grosseto & 220,785 & 218,538 & 396 & 3708 & 1.18 & 28 & 73 & 12.7 & 33.2 & 102 & 46.4 \\
\hline Livorno & 333,509 & 329,590 & 477 & 8079 & 0.56 & 62 & 195 & 18.6 & 58.8 & 260 & 78.4 \\
\hline Lucca & 388,678 & 380,676 & 1351 & 11,010 & 0.42 & 151 & 192 & 38.8 & 49.9 & 347 & 90.2 \\
\hline $\begin{array}{l}\text { Massa and } \\
\text { Carrara }\end{array}$ & 193,934 & 189,841 & 1051 & 6442 & 0 & 153 & 179 & 78.9 & 93.3 & 336 & 175 \\
\hline Pisa & 422,310 & 416,425 & 930 & 15,667 & 1.55 & 91 & 341 & 21.5 & 81.3 & 433 & 103 \\
\hline Pistoia & 293,059 & 290,819 & 747 & 9640 & 0.96 & 76 & 199 & 25.9 & 68.2 & 275 & 94.2 \\
\hline Prato & 258,152 & 256,047 & 532 & 9790 & 1.02 & 47 & 203 & 18.2 & 79.0 & 250 & 97.2 \\
\hline Siena & 266,238 & 263,526 & 427 & 4450 & 2.17 & 32 & 102 & 12.0 & 38.5 & 134 & 50.6 \\
\hline Umbria & 880,285 & 865,013 & 1385 & 26,064 & 0.670 & 80 & 530 & 9.10 & 60.7 & 610 & 69.9 \\
\hline Perugia & 655,403 & 643,311 & 1008 & 19,843 & 0.71 & 51 & 369 & 7.80 & 56.8 & 420 & 64.7 \\
\hline Terni & 224,882 & 221,702 & 377 & 6221 & 0.55 & 29 & 161 & 12.9 & 72.1 & 190 & 85.1 \\
\hline Marches & $1,518,400$ & $1,501,406$ & 6549 & 33,194 & 2.59 & 987 & 720 & 65.0 & 47.7 & 1709 & 113 \\
\hline Ancona & 469,750 & 465,023 & 1875 & 9711 & 2.16 & 218 & 185 & 46.4 & 39.6 & 403 & 86.2 \\
\hline $\begin{array}{l}\text { Ascoli } \\
\text { Piceno }\end{array}$ & 206,363 & 204,575 & 290 & 4790 & 4.95 & 12 & 125 & 5.8 & 60.8 & 137 & 66.7 \\
\hline Fermo & 173,004 & 170,248 & 473 & 4337 & 2.16 & 67 & 69 & 38.7 & 40.2 & 137 & 79.8 \\
\hline Macerata & 312,146 & 307,421 & 1154 & 7851 & 2.16 & 145 & 159 & 46.5 & 51.3 & 305 & 98.5 \\
\hline $\begin{array}{l}\text { Pesaro and } \\
\text { Urbino }\end{array}$ & 357,137 & 354,139 & 2757 & 6505 & 4.95 & 545 & 182 & 153 & 51.2 & 727 & 204 \\
\hline Lazio & $5,865,544$ & $5,720,796$ & 8010 & 148,533 & 1.00 & 863 & 2815 & 14.7 & 48.6 & 3717 & 64.2 \\
\hline
\end{tabular}


Table 1 Number of SARS-CoV-2 cases, COVID-19 deaths and COVID-19 mortality rates (deaths/100,000/wave timeframe) in the 1st and 2 nd waves in 2020 divided by province (Continued)

\begin{tabular}{|c|c|c|c|c|c|c|c|c|c|c|c|}
\hline $\begin{array}{l}\text { Province/ } \\
\text { Region }\end{array}$ & $\begin{array}{l}\text { Population } \\
\text { Jan 1, } 2020\end{array}$ & $\begin{array}{l}\text { Population } \\
\text { Jan 1, } 2021\end{array}$ & $\begin{array}{l}\text { Cases } \\
1 \text { st } \\
\text { wave }\end{array}$ & $\begin{array}{l}\text { Cases } \\
\text { 2nd } \\
\text { wave }\end{array}$ & $\begin{array}{l}\text { Seroprev. } \\
\text { (\%) }\end{array}$ & $\begin{array}{l}\text { Deaths } \\
\text { 1st wave }\end{array}$ & $\begin{array}{l}\text { Deaths } \\
\text { 2nd } \\
\text { wave }\end{array}$ & $\begin{array}{l}\text { Mortality } \\
\text { 1st wave }\end{array}$ & $\begin{array}{l}\text { Mortality } \\
\text { 2nd wave }\end{array}$ & $\begin{array}{l}\text { All } \\
\text { Deaths }\end{array}$ & $\begin{array}{l}\text { Overall } \\
\text { Mortality }\end{array}$ \\
\hline Frosinone & 485,241 & 473,467 & 663 & 12,990 & 0.19 & 79 & 162 & 16.3 & 33.8 & 241 & 50.3 \\
\hline Latina & 576,655 & 561,139 & 607 & 13,625 & 0.50 & 44 & 294 & 7.6 & 51.7 & 340 & 59.8 \\
\hline Rieti & 154,232 & 151,668 & 411 & 4565 & 3.00 & 41 & 149 & 26.6 & 97.4 & 191 & 125 \\
\hline Rome & $4,333,274$ & $4,227,588$ & 5872 & 108,988 & 1.05 & 672 & 2016 & 15.5 & 47.1 & 2724 & 63.6 \\
\hline Viterbo & 316,142 & 306,934 & 457 & 8365 & 1.52 & 27 & 194 & 8.5 & 62.3 & 221 & 70.9 \\
\hline Abruzzo & $1,305,770$ & $1,285,256$ & 3261 & 31,124 & 1.29 & 461 & 794 & 35.3 & 61.3 & 1264 & 97.6 \\
\hline Chieti & 383,189 & 376,397 & 818 & 6284 & 1.40 & 131 & 136 & 34.2 & 35.8 & 270 & 71.1 \\
\hline L'Aquila & 296,491 & 292,356 & 225 & 10,604 & 0.54 & 11 & 350 & 3.7 & 119 & 362 & 123 \\
\hline Pescara & 318,678 & 314,689 & 1586 & 5447 & 1.69 & 239 & 116 & 75.0 & 36.6 & 360 & 114 \\
\hline Teramo & 307,412 & 301,814 & 632 & 8789 & 1.48 & 80 & 192 & 26.0 & 63.0 & 272 & 89.3 \\
\hline Molise & 302,265 & 296,547 & 426 & 5971 & 0.81 & 28 & 175 & 9.3 & 58.4 & 203 & 67.8 \\
\hline Campobasso & 218,679 & 214,629 & 364 & 3829 & 0.66 & 22 & 110 & 10.1 & 50.8 & 132 & 60.9 \\
\hline Isernia & 83,586 & 81,918 & 62 & 2142 & 1.19 & 6 & 65 & 7.2 & 78.5 & 71 & 85.8 \\
\hline Campania & $5,785,861$ & $5,679,759$ & 4648 & 182,462 & 0.89 & 517 & 2915 & 8.9 & 50.8 & 3447 & 60.1 \\
\hline Avellino & 413,926 & 405,963 & 552 & 8289 & 0 & 62 & 143 & 15.0 & 34.9 & 206 & 50.3 \\
\hline Benevento & 274,080 & 269,233 & 209 & 4423 & 0 & 19 & 137 & 6.9 & 50.4 & 156 & 57.4 \\
\hline Caserta & 922,171 & 911,606 & 543 & 33,741 & 1.48 & 53 & 540 & 5.7 & 58.9 & 596 & 65.0 \\
\hline Naples & $3,082,905$ & $3,017,658$ & 2652 & 111,294 & 1.04 & 314 & 1811 & 10.2 & 59.4 & 2133 & 69.9 \\
\hline Salerno & $1,092,779$ & $1,075,299$ & 692 & 24,715 & 0.31 & 69 & 284 & 6.3 & 26.2 & 356 & 32.8 \\
\hline Apulia & $4,008,296$ & $3,926,931$ & 4502 & 84,951 & 0.88 & 566 & 2037 & 14.1 & 51.3 & 2614 & 65.9 \\
\hline Bari & $1,249,246$ & $1,222,818$ & 1491 & 33,237 & 1.50 & 153 & 636 & 12.2 & 51.5 & 793 & 64.2 \\
\hline $\begin{array}{l}\text { Barletta- } \\
\text { Andria-Trani }\end{array}$ & 388,390 & 382,685 & 380 & 10,058 & 0.77 & 66 & 295 & 17.0 & 76.5 & 361 & 93.6 \\
\hline Brindisi & 390,456 & 382,454 & 659 & 5795 & 0.85 & 67 & 100 & 17.2 & 25.9 & 167 & 43.2 \\
\hline Foggia & 616,310 & 601,419 & 1170 & 18,639 & 1.02 & 161 & 655 & 26.1 & 108 & 819 & 135 \\
\hline Lecce & 791,122 & 777,507 & 521 & 6420 & 0.01 & 85 & 114 & 10.7 & 14.5 & 200 & 25.5 \\
\hline Taranto & 572,772 & 560,048 & 281 & 10,802 & 0.67 & 34 & 237 & 5.9 & 41.8 & 274 & 48.4 \\
\hline Basilicata & 556,934 & 547,579 & 400 & 10,055 & 0.72 & 36 & 214 & 6.5 & 38.8 & 251 & 45.4 \\
\hline Potenza & 360,936 & 354,122 & 189 & 6739 & 0.83 & 27 & 156 & 7.5 & 43.6 & 184 & 51.5 \\
\hline Matera & 195,998 & 193,457 & 211 & 3316 & 0.50 & 9 & 58 & 4.6 & 29.8 & 67 & 34.4 \\
\hline Calabria & $1,924,701$ & $1,877,728$ & 1179 & 22,191 & 0.51 & 129 & 368 & 6.7 & 19.4 & 497 & 26.1 \\
\hline Catanzaro & 354,851 & 346,514 & 214 & 3134 & 0.40 & 31 & 49 & 8.7 & 14.0 & 80 & 22.8 \\
\hline Cosenza & 700,385 & 684,786 & 468 & 6676 & 0.78 & 48 & 176 & 6.9 & 25.4 & 224 & 32.3 \\
\hline Crotone & 170,718 & 166,617 & 119 & 2065 & 0.11 & 10 & 35 & 5.9 & 20.8 & 45 & 26.7 \\
\hline $\begin{array}{l}\text { Reggio di } \\
\text { Calabria }\end{array}$ & 541,278 & 526,586 & 294 & 8586 & 0.18 & 29 & 81 & 5.4 & 15.2 & 110 & 20.6 \\
\hline $\begin{array}{l}\text { Vibo } \\
\text { Valentia }\end{array}$ & 157,469 & 153,225 & 84 & 1730 & 1.12 & 11 & 27 & 7.0 & 17.4 & 38 & 24.5 \\
\hline Sicily & $4,968,410$ & $4,840,876$ & 3056 & 89,352 & 0.37 & 342 & 2390 & 6.9 & 48.7 & 2747 & 56.0 \\
\hline Agrigento & 429,611 & 419,847 & 135 & 3651 & 0.19 & 24 & 107 & 5.6 & 25.2 & 131 & 30.8 \\
\hline Caltanissetta & 260,779 & 252,803 & 186 & 3733 & 0 & 18 & 81 & 6.9 & 31.5 & 99 & 38.6 \\
\hline Catania & $1,104,974$ & $1,066,765$ & 779 & 26,464 & 0.26 & 103 & 849 & 9.3 & 78.2 & 957 & 88.1 \\
\hline Enna & 162,368 & 158,183 & 438 & 2866 & 0 & 34 & 76 & 20.9 & 47.4 & 110 & 68.6 \\
\hline
\end{tabular}


Table 1 Number of SARS-CoV-2 cases, COVID-19 deaths and COVID-19 mortality rates (deaths/100,000/wave timeframe) in the 1st and 2nd waves in 2020 divided by province (Continued)

\begin{tabular}{|c|c|c|c|c|c|c|c|c|c|c|c|}
\hline $\begin{array}{l}\text { Province/ } \\
\text { Region }\end{array}$ & $\begin{array}{l}\text { Population } \\
\text { Jan 1, } 2020\end{array}$ & $\begin{array}{l}\text { Population } \\
\text { Jan 1, } 2021\end{array}$ & $\begin{array}{l}\text { Cases } \\
1 \text { st } \\
\text { wave }\end{array}$ & $\begin{array}{l}\text { Cases } \\
\text { 2nd } \\
\text { wave }\end{array}$ & $\begin{array}{l}\text { Seroprev. } \\
\text { (\%) }\end{array}$ & $\begin{array}{l}\text { Deaths } \\
\text { 1st wave }\end{array}$ & $\begin{array}{l}\text { Deaths } \\
\text { 2nd } \\
\text { wave }\end{array}$ & $\begin{array}{l}\text { Mortality } \\
\text { 1st wave }\end{array}$ & $\begin{array}{l}\text { Mortality } \\
\text { 2nd wave }\end{array}$ & $\begin{array}{l}\text { All } \\
\text { Deaths }\end{array}$ & $\begin{array}{l}\text { Overall } \\
\text { Mortality }\end{array}$ \\
\hline Messina & 620,721 & 609,223 & 474 & 10,246 & 0.32 & 59 & 136 & 9.5 & 22.1 & 195 & 31.7 \\
\hline Palermo & $1,243,328$ & $1,214,291$ & 500 & 24,929 & 0.89 & 43 & 665 & 3.5 & 54.1 & 708 & 57.6 \\
\hline Ragusa & 321,215 & 314,950 & 87 & 6490 & 0.30 & 6 & 161 & 1.9 & 50.6 & 168 & 52.8 \\
\hline Siracusa & 397,037 & 386,451 & 321 & 5112 & 0.14 & 47 & 162 & 11.8 & 41.4 & 218 & 55.6 \\
\hline Trapani & 428,377 & 418,363 & 136 & 5861 & 0 & 8 & 153 & 1.9 & 36.1 & 161 & 38.0 \\
\hline Sardinia & $1,630,474$ & $1,598,225$ & 1366 & 28,920 & 0.50 & 145 & 712 & 8.9 & 44.1 & 858 & 53.1 \\
\hline Cagliari & 430,914 & 420,117 & 253 & 6573 & 0.38 & 19 & 161 & 4.4 & 37.8 & 180 & 42.3 \\
\hline Nuoro & 206,843 & 202,951 & 78 & 6055 & 0.24 & 12 & 123 & 5.8 & 60.0 & 135 & 65.9 \\
\hline Oristano & 156,078 & 153,226 & 61 & 2416 & 0.43 & 8 & 51 & 5.1 & 33.0 & 59 & 38.2 \\
\hline Sassari & 489,634 & 481,052 & 875 & 8997 & 0.78 & 90 & 247 & 18.4 & 50.9 & 338 & 69.6 \\
\hline $\begin{array}{l}\text { South } \\
\text { Sardinia }\end{array}$ & 347,005 & 340,879 & 99 & 4879 & 0.42 & 16 & 130 & 4.6 & 37.8 & 146 & 42.4 \\
\hline Italy & $60,244,639$ & $59,257,566$ & 235,839 & $1,808,260$ & 2.49 & 35,048 & 40,392 & 58.2 & 67.6 & 75,891 & 127 \\
\hline
\end{tabular}

100,000 persons and generally lower than the regional or national averages. The provinces with the highest first wave mortality, namely Piacenza (with the overall highest mortality in Italy with 333/100,000 persons), Cremona, Lodi and Bergamo, experienced the highest absolute decrease in mortality rates.

In the spline analysis, the relation between mortality in the first and second wave was U-shaped (Fig. 2), with a direct association between the estimates of the two waves up to 88 deaths $/ 100,000$, and above that an inverse pattern, with low second wave figures for those provinces with the highest rates during the first wave (88 through 192 deaths/100,000). The latter corresponded to a mortality rate of $22 / 100,000 /$ month, as only 5 deaths were recorded in February 2020 following the first disease diagnosis in Italian residents on February 20 . Crude analysis, ignoring control of potential confounders such as proportion elderly, living alone, and degree of mobility, gave similar results. When we repeated the main analysis by limiting the second wave to September-October 2020, i.e. by removing the November-December period when region-specific public health

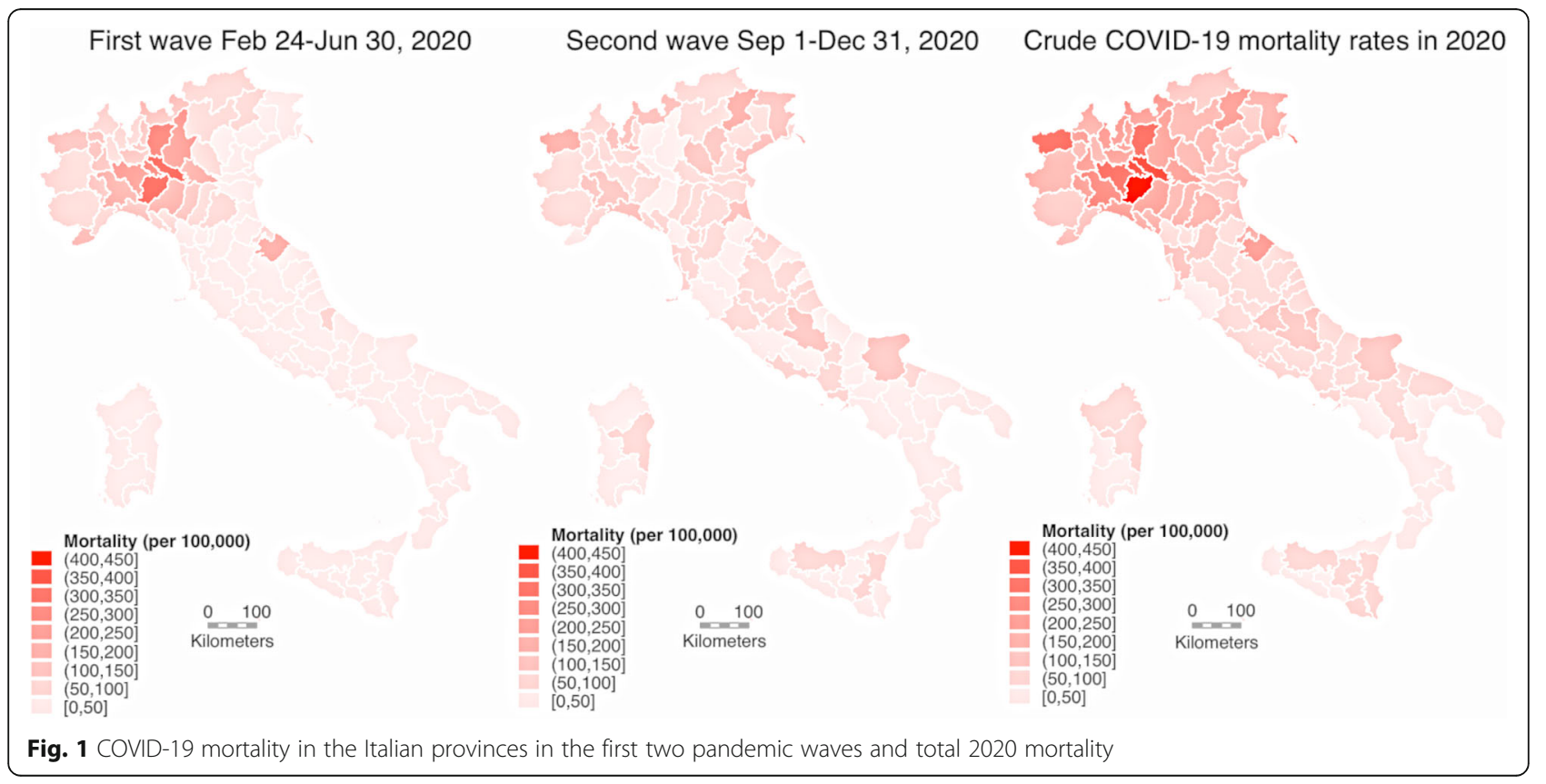



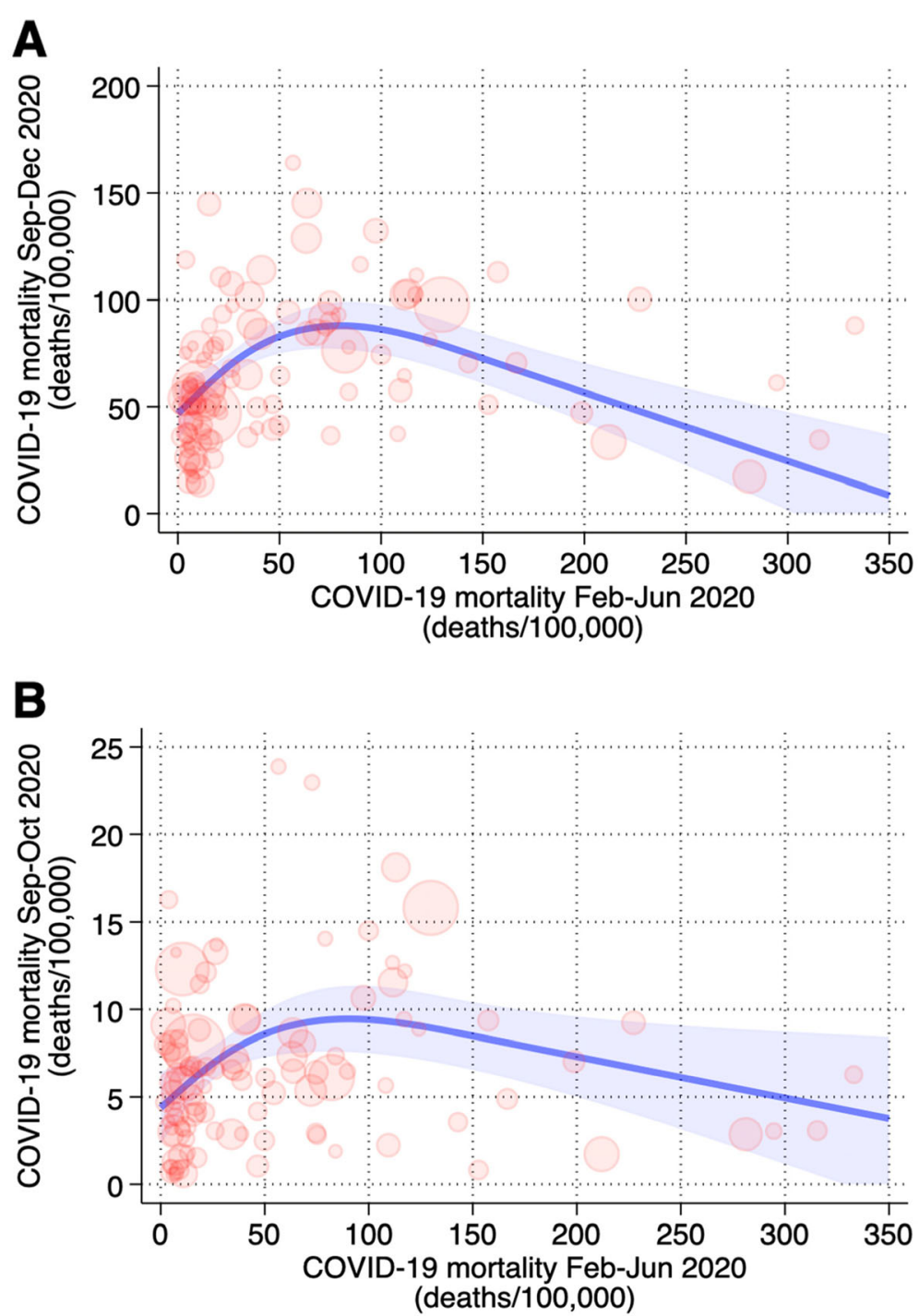

Fig. 2 Population-weighted cubic spline regression analysis of the relation between first (from February 24 to June 30, 2020) and (A) second wave (from September 1 to December 31, 2020) mortality rates of SARS-CoV-2 infection in Italian provinces or (B) second wave before the beginning of mobility restrictions (from September 1 to October 31, 2020) mortality rates of SARS-CoV-2 infection in Italian provinces adjusted for aging index, percentage commuting outside the municipality of residence on a daily basis, and percentage of dwellings occupied by only one resident. Shadow area is the $95 \%$ confidence interval of the predicted mean mortality rate (solid line); circles are province-specific values weighted for population size

and social distancing policies were first allowed and implemented, results were not substantially changed, though mortality in this truncated period was much lower (Fig. 2).

\section{Discussion}

Italy was the first country where the SARS-CoV-2 infection swept out of control, following the initial outbreak in China, where it was kept under control and geographically confined. The early arrival of the pandemic in Italy allowed a longer time frame to monitor and study the behavior of the epidemic. Italy did not allow the adoption of area-specific policies to curtail the outbreak until
November, using instead a nationwide approach imposed by the Italian Government. It has been suggested recently that severity of COVID-19 pneumonia in hospitalized subjects was lower during the second wave [26], possibly due to SARS-CoV-2 genomic variation [27]. However, during the time frame of this study there was no evidence of spread of SARS-CoV-2 variants [17], thus avoiding any confounding effect from differential geographic spread of viral lineages with different transmission or virulence features. In addition, recent assessment of fatality rates between first and second waves in Italy showed that they were comparable [28]. Nonetheless, we cannot rule out that improvement in COVID-19 
treatment may have decreased mortality during the pandemic, especially in the second wave, despite the lack of fully effective treatments [29-31]. Finally, vaccination had no role in curbing either of the two waves analyzed here, since almost no vaccine doses were administered in Italy in 2020. Indeed, the vaccination campaign had not begun yet in the investigated period, apart from a handful of 'demonstration' vaccinations administered to health professionals on December 27, 2020 at the national hub of the Spallanzani Hospital in Rome. Large scale administration started in January 2021 [32].

In contrast to our earlier analysis that relied on SARSCoV-2 infection incidence, when area-specific mortality was still unavailable, we used mortality from COVID-19 to gauge the severity of the waves.

Mortality is a far more reliable indicator than incidence for documenting the actual trends of the pandemic, because the detection and notification of newlyinfected cases from the general population depends on the availability and implementation of molecular testing, which in turn depends on health authority guidelines and policies [33]. Therefore, the number and type of individuals to be subject to SARS-CoV-2 testing may differ considerably across geographic areas or time periods independently of real differences in incidence, due to changing referral guidelines (such as the presence of COVID-19 symptoms) and availability of molecular tests. In Italy, first wave health policy allowed COVID19 molecular testing only for COVID-19 symptomatic individuals, while in the second wave much more testing occurred, with systematic extension to asymptomatic or weakly symptomatic individuals and therefore a great increase in number of notifications. As a consequence, the 'official' SARS-CoV-2 infection incidence strongly increased in the second wave. This increase, however, could have been an artifact due to the aforementioned changes in testing policy and availability, especially in light of the roughly comparable numbers of deaths in the two waves. However, incidence is less reliable compared to mortality in documenting the real trend of the pandemic, also due to its inherent differences compared with SARS-CoV-2 infection incidence and with the hybrid pattern of the related assessments at the population level, due to changes in molecular testing availability and policy.

We were able to confirm the previously reported incidence patterns for SARS-CoV-2 in Italy during 2020 [12]. The key findings were a positive association between the two waves when the epidemic was not particularly strong in the first part of the year, and a negative association when the first wave was severe. This finding was similar when using a different time frame for the second wave to account for the possible influence of local policies. Local policies were permitted beginning as of November 6, 2020. In addition, the pattern we investigated is based on 'real' data, compared to studies presenting predictive modeling [34, 35], thus strengthening our findings.

Provinces that were relatively lightly affected by the first wave had a similar experience in the second wave, with rates closely mirroring those experienced during the first phase of the outbreak. In contrast, the Italian provinces severely hit by the first wave, such as Bergamo, Lodi, Cremona and Piacenza, experienced a marked reduction of mortality in the second wave, and presumably in the spread of COVID-19. The reasons for such an inverse pattern, however, were not directly identifiable through this study and may only be hypothesized. One possibility is a higher likelihood of immunity for superspreaders and individuals with high mobility and propensity to transmission during the first wave [36]. Another possible explanation is the development of an additional cellular immunity against SARS-CoV-2 in the most severely hit population during the first wave $[37,38]$. The provinces most severely affected by the first wave showed a population-based seroprevalence in the $10-25 \%$ range, far below the estimated threshold for herd immunity of $50-70 \%$ [39]. It is possible that the prevalence of immunity, despite being below the herd immunity threshold, was high enough in provinces with a severe first wave to interact with behavioral factors and lessen the intensity of the second wave.

\section{Acknowledgements \\ not applicable.}

\section{Authors' contributions}

MV and TF designed the original study, and with KJR analyzed and interpreted the data, and wrote the manuscript. NO designed and carried out data analysis with TF and SDF. All authors read and approved the final manuscript.

\section{Funding}

This study was supported by the grants 'UNIMORE FAR 2019 and 2020 Interdisciplinare' of the Fondazione di Modena' to Dr. Vinceti and Dr. Filippini and by the FISR 2020-COVID19 grant by the Italian Ministry of the University and the Research to Dr. Vinceti.

\section{Availability of data and materials}

We used data made freely available from public sources. All data generated or analysed during this study are included in this published article.

\section{Declarations}

Ethics approval and consent to participate not applicable.

\section{Consent for publication}

not applicable.

\section{Competing interests}

The authors declare that they have no competing interests.

\section{Author details}

${ }^{1}$ Environmental, Genetic and Nutritional Epidemiology Research Center (CREAGEN), Section of Public Health, Department of Biomedical, Metabolic 
and Neural Sciences, University of Modena and Reggio Emilia, Modena, Italy. ${ }^{2}$ Department of Epidemiology, Boston University School of Public Health, Boston, MA, US. ${ }^{3}$ RTI Health Solutions, Research Triangle Park, Raleigh, NC, US. ${ }^{4}$ Department of Global Public Health, Karolinska Institutet, Stockholm, Sweden.

Received: 27 June 2021 Accepted: 29 October 2021

Published online: 11 November 2021

\section{References}

1. Kault D. Superspreaders, asymptomatics and COVID-19 elimination. Med J Aust. 2020;213(10):447-8 e1. https://doi.org/10.5694/mja2.50835.

2. Xia Y, Zhong L, Tan J, Zhang Z, Lyu J, Chen Y, et al. How to understand "herd immunity" in COVID-19 pandemic. Front Cell Dev Biol. 2020;8:547314. https://doi.org/10.3389/fcell.2020.547314.

3. Okell LC, Verity R, Watson OJ, Mishra S, Walker P, Whittaker C, et al. Have deaths from COVID-19 in Europe plateaued due to herd immunity? Lancet. 2020;395(10241):e110-e1. https://doi.org/10.1016/S0140-6736(20)31357-X.

4. Randolph HE, Barreiro LB. Herd immunity: understanding COVID-19. Immunity. 2020;52(5):737-41. https://doi.org/10.1016/j.immuni.2020.04.012.

5. Kwok KO, Lai F, Wei WI, Wong SYS, Tang JWT. Herd immunity - estimating the level required to halt the COVID-19 epidemics in affected countries. J Inf Secur. 2020;80(6):e32-e3. https://doi.org/10.1016/j.jinf.2020.03.027.

6. Omer SB, Yildirim I, Forman HP. Herd immunity and implications for SARSCoV-2 control. JAMA. 2020;324(20):2095-6. https://doi.org/10.1001/jama.202 0.20892 .

7. Papachristodoulou E, Kakoullis L, Parperis K, Panos G. Long-term and herd immunity against SARS-CoV-2: implications from current and past knowledge. Pathog Dis. 2020;78:ftaa025. https://doi.org/10.1093/femspd/ftaa 025.

8. Marques M, Rovira J, Nadal M, Domingo JL. Effects of air pollution on the potential transmission and mortality of COVID-19: a preliminary case-study in Tarragona Province (Catalonia, Spain). Environ Res. 2021;192:110315. https://doi.org/10.1016/j.envres.2020.110315.

9. Filippini T, Rothman KJ, Cocchio S, Narne E, Mantoan D, Saia M, et al. Associations between mortality from COVID-19 in two Italian regions and outdoor air pollution as assessed through tropospheric nitrogen dioxide. Sci Total Environ. 2021;760:143355. https://doi.org/10.1016/j.scitotenv.2020.1433 55.

10. Cherrie M, Clemens T, Colandrea C, Feng Z, Webb DJ, Weller RB, et al. Ultraviolet a radiation and COVID-19 deaths in the USA with replication studies in England and Italy. Br J Dermatol. 2021;185(2):363-70. https://doi. org/10.1111/bjd.20093.

11. Filippini T, Rothman KJ, Goffi A, Ferrari F, Maffeis G, Orsini N, et al. Satellitedetected tropospheric nitrogen dioxide and spread of SARS-CoV-2 infection in northern Italy. Sci Total Environ. 2020;739:140278. https://doi.org/10.1016/ j.scitotenv.2020.140278

12. Vinceti M, Filippini T, Rothman KJ, Di Federico S, Orsini N. SARS-CoV-2 infection incidence during the first and second COVID-19 waves in Italy. Environ Res. 2021;197:111097. https://doi.org/10.1016/j.envres.2021.111097.

13. Romagnani P, Gnone G, Guzzi F, Negrini S, Guastalla A, Annunziato F, et al. The COVID-19 infection: lessons from the Italian experience. J Public Health Policy. 2020;41(3):238-44. https://doi.org/10.1057/s41271-020-00229-y.

14. Presidente del Consiglio dei Ministri. DPCM 6 Novembre 2020. 2020 http:// www.governo.it/sites/new.governo.it/files/dpcm_20200322.pdf [Accessed August 30, 2021].

15. Vinceti M, Filippini T, Rothman KJ, Ferrari F, Goffi A, Maffeis G, et al. Lockdown timing and efficacy in controlling COVID-19 using mobile phone tracking. EClinicalMedicine. 2020;25:100457. https://doi.org/10.1016/j.eclinm.2 020.100457

16. ISTAT. COVID-19 deaths in Italy. Report March 5, 2021. https://www.istat.it/it/ files//2020/03/tabella-decessi-provinciali_fonte_istat_decessi_provinciali_ per_covid_fonte_ISS_5marzo.xlsx [Accessed August 30, 2021].

17. ELIXIR-IT. SARS-CoV-2 lineages of potential epidemiological interest in Italy. 2021 https://www.covid19dataportal.it/ [Accessed August 30, 2021].

18. Ministry of Health. Anti-COVID vaccination campaign. 2021 www.salute.gov. it [Accessed August 30, 2021].

19. ISTAT. Populations and households. Italian National Institute of Statistics. 2020 https://www.istat.it/en/population-and-households [Accessed August 31, 2021].
20. ISTAT. First results of the seroprevalence investigation on SARS-COV-2. Italian National Institute of Statistics. 2020 https://www.istat.it/it/files//2020/ 08/ReportPrimiRisultatilndagineSiero.pdf [Accessed August 30, 2021].

21. Harrell FE. Regression modeling strategies: with applications to linear models, logistic regression, and survival analysis. New York: Springer; 2001. https://doi.org/10.1007/978-1-4757-3462-1.

22. Filippini T, Adani G, Malavolti M, Garuti C, Cilloni S, Vinceti G, et al. Dietary habits and risk of early-onset dementia in an Italian case-control study. Nutrients. 2020;12(12):3682. https://doi.org/10.3390/nu12123682.

23. Filippini T, Mandrioli J, Malagoli C, Costanzini S, Cherubini A, Maffeis G, et al. Risk of amyotrophic lateral sclerosis and exposure to particulate matter from vehicular traffic: a case-control study. Int J Environ Res Public Health. 2021; 18(3):973. https://doi.org/10.3390/ijerph18030973.

24. Vinceti M, Rothman KJ, Crespi CM, Sterni A, Cherubini A, Guerra L, et al. Leukemia risk in children exposed to benzene and PM10 from vehicular traffic: a case-control study in an Italian population. Eur J Epidemiol. 2012; 27(10):781-90. https://doi.org/10.1007/s10654-012-9727-1.

25. Orsini N. XBRCSPLINE: Stata module to tabulate differences in predicted responses after restricted cubic spline models: Boston College Department of economics; 2009.

26. Borghesi A, Golemi S, Carapella N, Zigliani A, Farina D, Maroldi R. Lombardy, northern Italy: COVID-19 second wave less severe and deadly than the first? A preliminary investigation. Infect Dis (Lond). 2021;53(5):370-5. https://doi. org/10.1080/23744235.2021.1884745.

27. Toyoshima Y, Nemoto K, Matsumoto S, Nakamura Y, Kiyotani K. SARS-CoV-2 genomic variations associated with mortality rate of COVID-19. J Hum Genet. 2020;65(12):1075-82. https://doi.org/10.1038/s10038-020-0808-9.

28. Filippini T, Zagnoli F, Bosi M, Giannone ME, Marchesi C, Vinceti M. An assessment of case-fatality and infection-fatality rates of first and second COVID-19 waves in Italy: COVID-19 fatality rate in Italy. Acta Biomed. 2021; 92:e2021420. https://doi.org/10.23750/abm.v92iS6.12241.

29. COVID-19 RISK and Treatments (CORIST) Collaboration. Use of hydroxychloroquine in hospitalised COVID-19 patients is associated with reduced mortality: findings from the observational multicentre Italian CORIST study. Eur J Intern Med. 2020;82:38-47. https://doi.org/10.1016/j. ejim.2020.08.019.

30. Docea AO, Tsatsakis A, Albulescu D, Cristea O, Zlatian O, Vinceti M, et al. A new threat from an old enemy: reemergence of coronavirus (review). Int J Mol Med. 2020;45:1631-43. https://doi.org/10.3892/ijmm.2020.4555.

31. Di Castelnuovo A, Costanzo S, Antinori A, Berselli N, Blandi L, Bonaccio M, et al. Lopinavir/ritonavir and darunavir/cobicistat in hospitalized COVID-19 patients: findings from the multicenter Italian CORIST study. Front Med (Lausanne). 2021:8:639970. https://doi.org/10.3389/fmed.2021.639970.

32. CPD - Italian Civil Protection Department. Anti COVID-19 Vaccine Report. 2021 https:/italia.github.io/covid19-dashboard-vaccini/ [Accessed August 30, 2021].

33. De Matteis A, Turkmen Ceylan FB, Adrianopoli M, Ertek S. The contribution of testing in the fight against COVID-19: evidence from Italy. Eur J Pub Health. 2021;31(4):913-7. https://doi.org/10.1093/eurpub/ckab064.

34. Qian Y, Xie W, Zhao J, Xue M, Liu S, Wang L, et al. Investigating the effectiveness of re-opening policies before vaccination during a pandemic: SD modelling research based on COVID-19 in Wuhan. BMC Public Health. 2021;21(1):1638. https://doi.org/10.1186/s12889-021-11631-w.

35. Ahmed DA, Ansari AR, Imran M, Dingle K, Bonsall MB. Mechanistic modelling of COVID-19 and the impact of lockdowns on a short-time scale. PLoS One. 2021;16(10):e0258084. https://doi.org/10.1371/journal.pone.0258084.

36. Signorelli C, Zucchi A, Tersalvi CA, Ciampichini R, Beato E, Balzarini F, et al. High seroprevalence of SARS-CoV-2 in Bergamo: evidence for herd immunity or reason to be cautious? Int J Public Health. 2020;65(9):1815-7. https://doi.org/10.1007/s00038-020-01524-x.

37. Braun J, Loyal L, Frentsch M, Wendisch D, Georg P, Kurth F, et al. SARS-CoV2-reactive T cells in healthy donors and patients with COVID-19. Nature. 2020;587(7833):270-4. https://doi.org/10.1038/s41586-020-2598-9.

38. Mateus J, Grifoni A, Tarke A, Sidney J, Ramirez SI, Dan JM, et al. Selective and cross-reactive SARS-CoV-2 T cell epitopes in unexposed humans. Science. 2020;370(6512):89-94. https://doi.org/10.1126/science.abd3871.

39. Fontanet A, Cauchemez S. COVID-19 herd immunity: where are we? Nat Rev Immunol. 2020;20(10):583-4. https://doi.org/10.1038/s41577-020-00451-5.

\section{Publisher's Note}

Springer Nature remains neutral with regard to jurisdictional claims in published maps and institutional affiliations. 